\title{
ACUTE MYOCARDITIS IN THE MAGNETIC RESONANCE IMAGING ERA: PATIENT'S CHARACTERISTICS AND MID-TERM FOLLOW-UP
}

\author{
Carma Karam MD, Cardiology Department, Reference Cen- \\ ter of Cardiovascular Disease, Hospital Am- \\ broise Paré, AP-HP Boulogne-Billancourt, \\ Université de Versailles-Saint Quentin en Yve- \\ lines, France. \\ Ali Alghamdi MD, Cardiology Department, Reference Cen- \\ ter of Cardiovascular Disease, Hospital Am- \\ broise Paré, AP-HP Boulogne-Billancourt, \\ Université de Versailles-Saint Quentin en Yve- \\ lines, France. \\ Nicolas Mansencal MD, Cardiology Department, Reference Cen- \\ ter of Cardiovascular Disease, Hospital Am- \\ broise Paré, AP-HP Boulogne-Billancourt, \\ Université de Versailles-Saint Quentin en Yve- \\ lines, France. \\ Stephen Binsse MD, Radiology Department, Hospital Ambroi- \\ se Paré, AP-HP Boulogne-Billancourt, Univer- \\ sité de Versailles-Saint Quentin en Yvelines, \\ France. \\ Pascal Lacombe $\quad$ MD, Radiology Department, Hospital Ambroise \\ Paré, AP-HP Boulogne-Billancourt, Université \\ de Versailles-Saint Quentin en Yvelines, France. \\ Olivier Dubourg MD, Cardiology Department, Reference Cen- \\ ter of Cardiovascular Disease, Hospital Am- \\ broise Paré, AP-HP Boulogne-Billancourt, \\ Université de Versailles-Saint Quentin en Yve- \\ lines, France.
}

Corresponding author

Carma Karam

E-mail: carmakaram@hotmail.com

\begin{abstract}
Background: Myocarditis is an inflammation of myocardial tissue that presents with a wide range of symptoms. Cardiovascular magnetic resonance (CMR) has become the first choice of non-invasive assessment of myocardial inflammation in suspected pts. Aims: The aim of this study was to report
\end{abstract}


clinical, paraclinical and follow up data observed in pts with acute myocarditis confirmed by CMR in a single center. Methods: We retrospectively studied 27 pts admitted for acute myocarditis between November 2010 and November 2012. All pts had ECG, echocardiography and CMR. Ultrasensitive cardiac troponin and CRP were measured. Coronary angiogram was performed in case of acute myocardial infarction-like syndrome or in the presence of CV risk factors. We reviewed the files of the hospital out-patient clinic and contacted the pts or their cardiologists by phone for those followed outside the hospital. Results: There were 23 males $(85.2 \%)$ and 4 females, aged $36 \pm 19$ yrs. ST elevation was found in 17 pts (62.9\%). All had elevated cardiac troponin. Echocardiography showed abnormalities of wall motion in 16 pts (59.2\%). Mean LVEF on CMR was $53.96 \pm 9.9 \%$ and late gadolinium enhancement was in lateral in $80 \%$, in inferior in $10 \%$ and anterior or apical wall in $10 \%$. Coronary angiogram was normal, performed in 14 pts (51.8\%). Complications included VT in 4 pts (14.8\%), AF in 2, and cardiac tamponade in 1. Follow-up was obtained for 23 pts (85\%). One died for pulmonary embolism on lung cancer. All others had a favorable evolution. Conclusion: Our study showed that myocarditis affects in majority young and male patients. CMR appears as the main modality of diagnosis. Coronary angiogram is mandatory in case of $\mathrm{CV}$ risk factors and/or myocardial infarction-like presentation. Evolution is often favorable. Optimal medical therapy is still to be defined. Pts can be considered as cured in the absence of chest pain and in case of normalization of echocardiography and/or CMR at follow-up.

Keywords: Myocarditis; Cardiovascular magnetic resonance.

\section{BACKGROUND}

Myocarditis is a cardiac disease associated with inflammation and injury of the myocardium. ${ }^{(1)}$ Acute myocarditis is frequently diagnosed as non ischemic dilated cardiomyopathy with symptoms that have been present for a few weeks to several months. However manifestations range from subclinical disease to sudden death, with new-onset atrial or ventricular arrythmias, complete heart block or an acute myocardial infarction-like syndrome. Hence, the diagnosis of myocarditis based on the clinical presentation is usually not possible. Myocarditis has been reported in up to $12 \%$ of young adults presenting with sudden death. ${ }^{(2,3,4,5)}$ The incidence of non-fatal myocarditis is likely higher than actually diagnosed, mostly due to the challenges of establishing the diagnosis in standard clinical settings. Cardiac symptoms are variable and may include precordial chest pain, fatigue, palpitations, syncope and decreased exercise tolerance. Although a viral prodrome with fever, myalgia, and respiratory or gastrointestinal symptoms is classically associated with myocarditis, reported symptoms are highly variable. ${ }^{(6)}$

Infectious disease accounts for the majority of cases due to either a direct viral infection or post-viral immune-mediated reaction. However, myocardial inflammation may also be triggered by reversible and/or irreversible toxic, ischemic or mechanical injury, drug-related inflammation, transplant rejection or other immune reactions.

Following the initial injury, cytokines and B cells are activated by local and systemic immune responses with subsequent edema, autoantibody production, and ad- 
ditional myocyte injury. Although the molecular and cellular pathophysiology may differ between different etiologies, cellular infiltration, oedema, necrosis and fibrotic scars are common features.

With the limited specificity of the history and the physical examination, patients may appear almost normal, may have non-specific symptoms, but may also present with features of acute myocardial infarction, or heart failure with hemodynamic compromise. Physical exams are often normal. ST segment elevation or T wave inversion are the most sensitive ECG criterion.

Depending on the severity and time of testing during the course of disease, serum biomarkers of myocardial injury may be elevated. Troponin I has high sensitivity $(89 \%)$ but limited specificity (34\%) in the diagnosis of myocarditis. ${ }^{(7)}$ Clinical and experimental data suggest that increase levels of cardiac troponin I are more common than increased levels of creatine kinase MB in acute myocarditis. ${ }^{(8)}$

In adults, the recommended indications for endomyocardial biopsy (EMB) are confined to patients with heart failure ${ }^{(9)}$ and therefore EMB is not recommended in many patients with myocarditis.

Ultrasound studies of the heart in myocarditis typically are performed to visualize associated functional abnormalities, wall thickness and pericardial effusion. ${ }^{(10,11)}$

Different from other diagnostic modalities, targets for CMR not only include functional and morphological abnormalities but also tissue pathology as diagnostic features of myocardial inflammation. Myocardial late gadolinium enhancement (LGE) specifically reflects irreversible myocardial injury, i.e. necrosis and fibrosis.

The prognosis of patients with myocarditis depends on clinical presentation and different clinical parameters.

\section{METHODS}

We retrospectively reviewed the files and charts of 27 patients diagnosed as acute myocarditis confirmed by CMR between November 2010 and November 2012 in the cardiology department, Ambroise Paré university hospital, Paris, France.

Diagnosis of acute myocarditis was based on the presence of LGE in subepicardium in all patients except one by the sequence of diffusion on CMR.

Data collected during the hospitalization included the clinical status, biological serum tests (CRP, ultrasensitive cardiac troponin), ECG, echocardiography, CMR and coronary angiogram if needed.

We compared the finding results of the different modalities used during the hospitalization i.e. ECG, echocardiography and CMR to search for matching or correlations. 
Patients follow up consisted in data collection for the complications, the evolution of the clinical symptoms, the results of investigations as follow-up echocardiography and CMR and the duration of the treatment. We reviewed the files of the out-patient clinic for the patients followed in the hospital and contacted the patients or their cardiologists by phone for those followed outside the hospital.

\section{RESULTS}

\section{In hospital patients characteristics}

Patient demographics are presented in Table 1. There were 23 males (85.2\%) and 4 females $(14.8 \%)$ with a mean age $36 \pm 19$ years (range $15-87$ years). All our patients complained of acute chest pain which was the main symptom to sight the medical emergency. Of those, 25 patients $(92.6 \%)$ were stable with a mean systolic blood pressure of $121 \mathrm{mmHg}$ and diastolic blood pressure of $70 \mathrm{mmHg}$. The two others presented with a low systolic blood pressure less than $90 \mathrm{~mm} \mathrm{Hg}$ of systolic pressure without other signs of shock or heart failure.

The presence of ST segment elevation on the initial ECG was found in 17 patients (62.9\%). All the patients had an elevated ultra-sensitive cardiac troponin with a mean of $11.5 \pm 10.9 \mu \mathrm{g} / \mathrm{l}$. CRP tested in 25 patients (92.6\%) was elevated in all the patients with a mean of $73.9 \pm 25.4 \mathrm{mg} / \mathrm{l}$.

Table 1 - Patients Demographics

\begin{tabular}{|c|c|}
\hline Patients & $(\mathbf{n}=\mathbf{2 7})$ \\
\hline Mean age, years & $36 \pm 19$ \\
\hline$<45$ yrs of age, $\%$ & 77.7 \\
\hline Male gender, \% & 85.2 \\
\hline Mean in-hospital stay, nights & $6.1 \pm 1.4$ \\
\hline Mean $\mathrm{SBP}^{\star}, \mathrm{mmHg}$ & 121 \\
\hline Mean $\mathrm{DBP}^{\dagger}, \mathrm{mmHg}$ & 70 \\
\hline ST segment elevation, $\%$ & 62.9 \\
\hline Elevated Troponin, mcg/L & $11.5 \pm 10.9$ \\
\hline Mean ejection fraction $\left(\mathrm{TTE}^{*}\right), \%$ & $55.5 \pm 7.3$ \\
\hline Average ejection fraction $\left(\mathrm{CMR}^{\S}\right), \%$ & $53.96 \pm 9.9$ \\
\hline
\end{tabular}

102 / Brazilian Journal of Medicine Health. 2013 Ago; 2(1):99-110 
Echocardiography was performed at baseline in all patients. It showed abnormalities of left ventricle regional function with hypokinesia or akinesia of wall motion in 16 patients $(59.2 \%)$. The mean ejection fraction of all patients was estimated at $55.5 \pm 7.3 \%$, range $35-65 \%$. There was pericardial effusion in 8 patients $(29.6 \%)$ that seemed to be independent of the presence of abnormalities of ejection fraction or regional motion of the left ventricle. CMR was performed for all patients confirming the diagnosis of the acute myocarditis with a mean ejection fraction of left ventricle estimated at $53.96 \pm 9.9 \%$, range $30-71 \%$. Table 2 summarizes CMR results.

Table 2 - Summary of the CMR results during the hospitalization

\begin{tabular}{ll}
\hline & $(\mathbf{n = 2 7})$ \\
\hline Mean EF* $\%$ & $53.96 \pm 9.9$ \\
Peric. Eff. Ass. ${ }^{* *}, \%$ & 22.2 \\
Regional wall affected of $\mathrm{LV}^{* *}, \%$ & \\
LAT & 48.1 \\
INF LAT & 18.5 \\
INF & 11.1 \\
LAT APIC & 7.4 \\
ANT APIC & 3.7 \\
ANT LAT & 3.7 \\
INF LAT SEPT & 3.7 \\
APIC & 3.7 \\
\hline
\end{tabular}

"Ejection fraction, ${ }^{\# *}$ Pericardial effusion associated,

" Regional wall affected of the left ventricle; LAT: lateral,

INF LAT: infero-lateral, INF: inferior, LAT APIC: latero-apical,

ANT APIC: antero-apical, ANT LAT: anterolateral,

INF LAT SEPT; infero-latero-septal, APIC: apical.

The majority of patients ( 22 patients $=81.4 \%$ ) showed that lateral wall of left ventricle is affected by the myocarditis in the CMR as shown in Table 2. Figures 1, 2 and 3 show the abnormalities in the left ventricle detected by CMR. 
Figure 1 - Short axis view shows LGE in sub-epicardium of the lateral wall of left ventricle indicated by the white arrow.

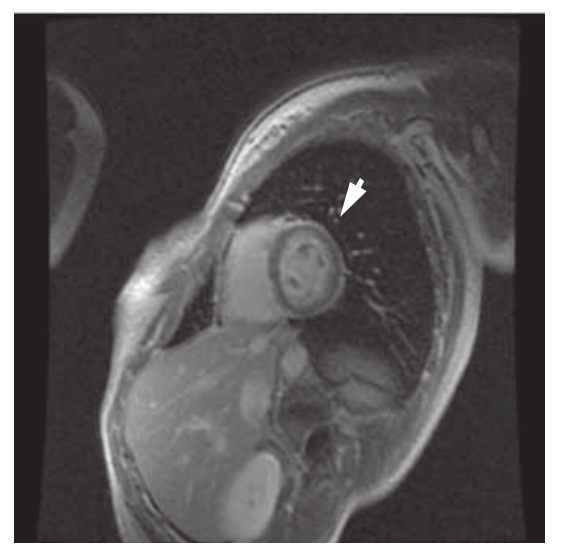

Figure $2-4$ chamber view shows LGE in sub-epicardium of the lateral wall of left ventricle indicated by the black arrow.

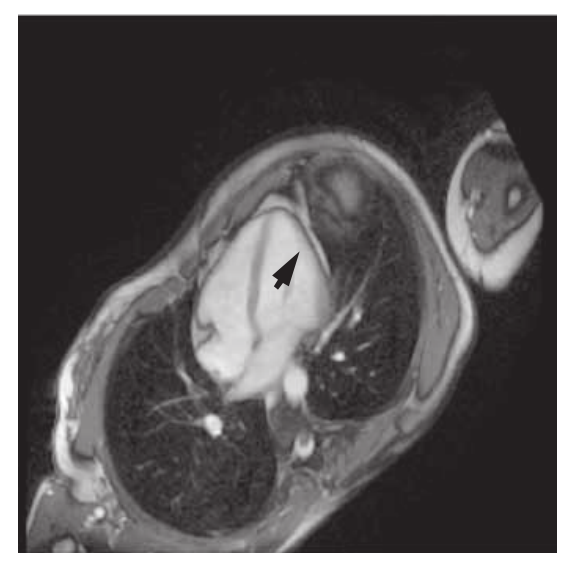

Figure 3 - Short axe view shows LGE in the sub-epicardium of the lateral wall of left ventricle (indicated by arrow n.1) associated with epicardial effusion (indicated by the arrow n.2).

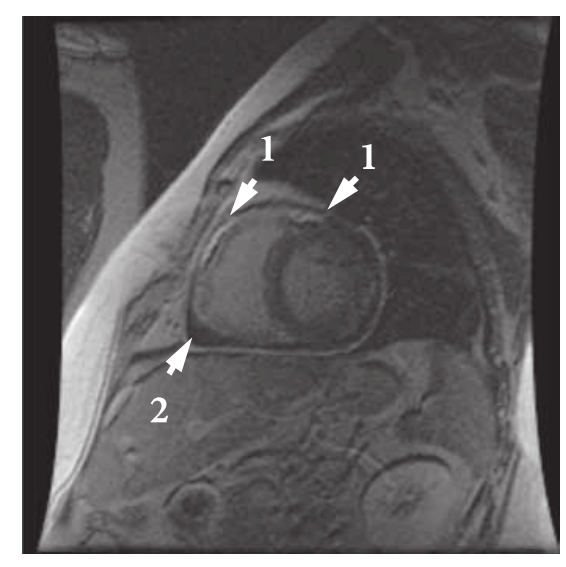

104 / Brazilian Journal of Medicine Health. 2013 Ago; 2(1):99-110 
Coronary angiogram was performed in 14 patients (51.8\%). The results showed no significant affect of the coronary system in all the patients and left ventricle angiogram was performed in 9 patients with a mean angiographic ejection fraction at $55 \pm 8 \%$.

All but one patient (26(96.3\%)) were treated by betablockers and angiotensin converting enzyme inhibitors or angiotensin-II receptor blockers. One young patient (20 years old) did not receive medical therapy because he had a very mild form of myocarditis.

We compared the findings of CMR and ECG for all patients to see if there was a matching between the regional wall of left ventricle affected by myocarditis diagnosed in CMR and with those in ECG represent ST segment elevation, we found that there is matching in 11 patients out of 17 patients (64.7\%). Comparison of regional wall motion dysfunction between CMR and echocardiography showed that there was a matching in 6 patients out of $16(37.5 \%)$.

In 11 patients (40.7\%), echocardiography showed a normal regional wall motion of the left ventricle and abnormalities in 16 patients (29.3\%).

By comparison between the results of the abnormal echocardiography and the CRM, we found that there was a match in 6 patients over $16(37.5 \%)$.

\section{In hospital events}

Non - sustained ventricular tachycardia was observed in 4 patients (14.8\%), including the two patients who presented hypotension at admission without signs of shock. Cardiac arrhythmia had a good response to the beta blockers.

One patient $(3.7 \%)$ presented a normal sinus rhythm in alternative with atrial fibrillation and flutter with few ventricular extrasystoles.

One patient (3.7\%) presented an atrial fibrillation and converted to normal sinus rhythm after receiving amiodarone.

\section{Follow - up}

We achieved to collect follow - up data for 23 patients (85.2\%) with a mean duration of $360 \pm 216$ days.

One patient died 1 year after the hospital discharge from pulmonary embolism and had recent history of lung cancer.

At one - year follow-up, there was improvement of the symptoms by disappearing of the chest pain in all patients.

First control echocardiography was requested for 19 patients (70.4\%) with a mean delay of $56 \pm 5$ days. The echocardiography of control showed improvement in 
the ejection fraction with a mean of $61.8 \pm 6.9 \%$ vs. a mean ejection fraction $55 \pm 1.4 \%$ of the echocardiography during the hospitalization for the same group of patients.

Second control echocardiography was requested for 3 patients $(15.8 \%$ of whom had first control echocardiography $=11.1 \%$ of all patients) with a mean delay of 147 days, showed no improvement regarding the ejection fraction in the first control echocardiography.

First control CMR was performed for 12 patients ( $44.4 \%$ ) with a mean delay of $98 \pm 3.5$ days, showed a mean ejection fraction $59 \pm 5.7 \%$ vs. a mean ejection fraction of $52 \pm 11 \%$ during the hospitalization for the same group of patients.

Second control CMR was performed for 3 patients out of 12 (25\% of whom had the first control CMR) with a mean delay of 163 days, showed a mean ejection fraction of $65 \pm 2 \%$.

The mean duration of the treatment by the betablockers and angiotensin converting enzyme inhibitors for 12 patients is $68 \pm 21$ days.

\section{Complications at follow-up}

None of the patients presented complications post - discharge except in one patient who was treated by oral anticoagulant (fluindione, Previscan ${ }^{\circledR}$ ) for the occurrence of atrial fibrillation during the hospitalization. He had aggravation of pericardial effusion complicated by cardiac tamponade, and he was readmitted 3 days after discharge in a cardiogenic shock with an echocardiographic ejection fraction of $30 \%$ and needed a subxyphoid surgical pericardiostomy for fluid drainage.

\section{DISCUSSION}

The objective of our study was to report our experience with acute myocarditis at the CMR era. We observed a large predominance of males with 23 patients (85.2\%) and of young age with 21 patients $(77.7 \%)$ were less than 45 years old.

The fulminant form which needs urgent mechanical circulatory assistance was excluded because CMR was not performed due to an urgent need for cardiac assistance or monitoring. Fortunately, most cases of myocarditis are mild as compared with the literature. ${ }^{(12,13,14)}$ We noticed the immediate good prognosis without serious complications in the majority of patients except in one who had the tamponade and was receiving oral anticoagulation for atrial fibrillation.

Aside from a low specificity, the presence of either ST segment elevation or T wave inversion as the most sensitive ECG criterion is present in less than $50 \%$ of patients, even during the first weeks of the disease. ${ }^{(15)}$ Our study showed $63 \%$ of patients 
had ST segment elevations and a $64.7 \%$ match with the CMR results for the regional wall affected by myocarditis.

The diagnostic value of echocardiography is limited by the fact that many patients with less severe myocarditis have a normal echocardiography and the highly variable echocardiography findings lack specificity as previously reported.(10)

Our study showed a mean ejection fraction of $55.5 \pm 7.3 \%$. Echocardiography was considered normal in 10 patients (37\%). Even in those with abnormal left ventricle regional function of wall motion in echocardiography (16 patients), 10 patients showed no matching with the findings of CRM.

Numerous studies have investigated the diagnostic utility of non-contrast and contrast-enhanced CMR in patients with myocarditis. ${ }^{(16,17,18)}$ Results have consistently shown the clinical feasibility and high diagnostic accuracy with different single technique or combined CMR protocols.

Contrast - enhancement CMR (CE- CMR) enables visualization of myocardial damage in patients with myocarditis after intravenous injection of gadolinium. Due to different wash - in and wash - out kinetics, areas with myocardial changes, such as scarring, fibrosis and edema, retain gadolinium for prolonged periods. This provides an opportunity to visualize areas of myocarditis defined by histopathology, with a report sensitivity of $100 \%$ and specificity of $90 \%{ }^{(19)}$ Presence of late gadolinium enhancement is reported in 44 to $95 \%$ of patients with myocarditis. ${ }^{(19,20)}$

According to contrast enhancement (CE) patterns, this technique is also capable of ruling out an ischemic cause in the differential diagnosis of myocarditis because $\mathrm{CE}$ patterns in the setting of ischemic infarction always include the subendocardial layer of the myocardium. ${ }^{(21)}$ Enhancement patterns in myocarditis generally exclude the subendocardium with the exception of eosinophilic myocarditis frequently involving the endomyocardium ${ }^{(22,23)}$ (Figures 1, 2 and 3).

Our study showed the presence of late gadolinium enhancement in 25 patients (92.6\%) with exclusion the subendocardium, absent in one patient ( $3.7 \%$ ) and doubt of presence in sub-epicardium in another one patient ( $3.7 \%)$.

We noticed that in 10 patients (37.1\%) the diagnosis of myocarditis was highly suspected based on clinical presentation, biology, ECG, echocardiography and coronary angiography before the confirmation by CMR while in 17 patients (62.9\%) those different modalities did not allow to suspect the diagnosis before CMR. Therefore, the CMR appears as an essential modality to achieve the diagnosis of myocarditis.

The indications for the coronary angiogram during the hospitalization were the presence of cardiovascular risk factors in 10 patients $(71.4 \%$ out of 14 the patients had coronary angiogram) and/or the suspicion of myocardial infarction in 10 patients 
(71.4\%). The mean age of the patients undergoing coronary angiogram was $42 \pm 17$ years ( $20-78$ years) vs $28 \pm 19$ years $(15-87$ years $)$ for those who did not.

For the one patient aged 51 who was readmitted 3 days after discharge for a cardiac tamponade, the diagnostic coronary angiogram was performed later, after stabilization of his status due to presence of cardiovascular risk factors.

The coronary angiogram was not performed for the remaining 12 patients (44.4\%) because of the young age in 10 patients ( $83.3 \%$ out of 12 patients) with a mean age $28 \pm 19$ years ( $15-87$ years), the absence of ischemic signs on ECG in 2 patients $(16.6 \%)$ and the presence of signs of acute myocarditis without endocardial damage on CMR in one old 87 years patient who had lung cancer (8.3\%).

\section{STUDY LIMITATIONS}

Our study did not include the fulminant or mild undiagnosed cases of myocarditis. We did not achieve to collect follow-up data for all patients. Four (14.8\%) were lost to follow-up) because of either loss of correct personal data in their files or because they moved to another unknown address.

\section{CONCLUSION}

This retrospectively study showed that myocarditis is a cardiovascular disease that affects in majority young and male patients. Except in fulminant forms, CMR appears as the main modality of diagnosis for acute myocarditis. Coronary angiogram is mandatory in case of cardiovascular risk factors and/or myocardial infarction-like presentation. Evolution shows that the majority of cases recover from the acute state when the diagnosis is established as fast as possible. Optimal medical therapy is still to be defined. We used beta blockers and angiotensin converting enzyme inhibitors or angiotensine receptor blockers in all except one patient. Patients can be considered as cured in the absence of chest pain and in case of normalization of echocardiography and/or CMR at follow-up.

\section{REFERENCES}

1. Caforio AL, Mahon NJ, Tona F, McKenna WJ. Circulating cardiac autoantibodies in dilated cardiomyopathy and myocarditis: pathogenetic and clinical significance. Eur J Heart Fail. 2002;4:411-7.

2. Fabre A, Shepparrd MN. Sudden adult death syndrome and other non-ischemic causes of sudden cardiac death. Heart. 2006; 92:316-20. [PubMed: 15923280]

3. Doolan A, Langlois N, Semsarian C. Causes of sudden cardiac death in young Australian. Med J Aust. 2004; 180:110-2. [PubMed: 14748671] 
4. Puranik R, Chow CK, Duflou JA, Kilborn MJ, McGuire MA. Sudden death in the young. Heart Rhythm. 2005;2:1277-82. [PubMed: 16360077]

5. Virmani R, Burke AP, Farb A. Sudden cardiac death. Cardiovasc Pathol. 2001;10:211-8. [PubMed: 11673058]

6. Magnani JW, Dec GW. Myocarditis : current trends in diagnosis and treatment. Circulation 2006;113:876-90.

7. Esfandiarei M, McManus BM. Molecular biology and pathogenesis of viral myocarditis. Annu Rev Pathol. 2008;3:127-55.

8. Caforio AL, Calabrese F, Angelini A,Tona F, Vinci A, Bottaro S. relevance of clinical and aetiopathogenetic features at diagnosis. Eur Heart J. 2007;28:1326-33.

9. Smith SC, Ladenson JH, Mason JW, Jaffe AS. Elevation of cardiac troponine I associated with myocarditis: experimental and clinical correlates. Circulation 1997;95:163-8.

10. Lauer B, Niederau C, Kül U et al. Cardiac troponin T in patients with clinically suspected myocarditis. J Am Coll Cardiol 1997;30:1354-9.

11. Cooper LT, Baughman KL, Feldman AM, Frustaci A, Jessup M, Kuhl U et al. American Heart Association, American College of Cardiology, European Society of Cardiology, Heart Failure Society of America, Heart Failure Association of the European Society of Cardiology. The role of endomyocardial biopsy in the management of cardiovascular disease: a scientific statement from the American Heart Association, the American College of Cardiology, and the European Society of Cardiology. Endorsed by the Heart Failure Society of America and the heart failure Association of the European Society of Cardiology. J Am Coll Cardiol 2007;50:1914-31. [PubMed; 17980265]

12. Pinamonti B, Alberti E, Cigalotto A, Dreas L, Salvi A, Silvestri F et al. Echocardiographic findings in myocarditis. Am-J-Cardiol 1988;62:258-91.[PubMed:3400607]

13. Felker GM, Boehmer JP, Hruban RH, Hutchins GM, Kasper EK, Baughman KL et al. Echocardiographic findings in fulminant and acute myocarditis. J Am Coll Cardiol 2000;36:227-32. [PubMed: 10898439]

14. D’Ambrosio A, Patti G, Manzoli A et al. The fate of acute myocarditis between spontaneous improvement and evolution to dilated cardiomyopathy: a review. Heart 2001;85:499-504.

15. Friman G, Wesslen L, Fohlman J, Karjalainen J, Rolf C. The epidemiology of infectious myocarditis , lymphocytic myocarditis and dilated cardiomyopathy. Eur Heart J 1995;16 Suppl O:36-41.

16. Karjalainen J, Heikkila J. Incidence of three presentations of acute myocarditis in young men in military service. A 20-year experience. Eur Heart J 1999;20:1120-5.

17. Morgera T, Di Lenarda A, Dreas L, Pinamonti B, Humar F, Bussani R et al. Electrocardiography of myocarditis revisited: clinical and prognostic significance of electrocardiographic changes. Am Heart J 1992;124:455-67. [PubMed: 1636589]

18. Laissy JP, Messin B, Varenne O, Iung B, Karila-Cohen, Schouman-Claeys E et al. MRI of acute myocarditis: a comprehensive approach based on various imaging sequences. Chest 2002;122:163848. [PubMed; 12426265]

19. Abdel-Aty H, Boye P, Zagrosek A, Wassmuth R, Kumar A, Messroghli et al. Diagnostic performance of cardiovascular magnetic resonance in patients with suspected acute myocarditis; comparison of different approaches. J Am Coll Cardiol 2005;45:1815-22.[PubMed; 15936612]

20. Gutberlet M, Spors B, Thoma T, Bertram H, Deneck T, Felix R et al. Suspected chronic myocarditis at cardiac MR: diagnostic accuracy and association with immunohistologically detected inflammation and viral persistence. Radiology 2008;246:401-9. [PubMed: 18180335] 
21. Mahrholdt H, Goedecke C, Wagner A, Meinhardt G, Athanasiasdis A, Vogelsberg H et al. Cardiovascular magnetic resonance assessment of human myocarditis: a comparison to histology and molecular pathology. Circulation. 2004;109:1250-8.

22. Mahrholdt H, Wagner A, Deluigi CC, Kispert E, Hager S, Meinhardt G et al. Presentation, pattern of myocardial damage, and clinical course of viral myocarditis. Circulation. 2006;114:1581-90.

23. Kadalie CT. [MRI in chronic myocarditis].Z Kardiol. 2005;94(suppl 4)IV/94-IV/96.

24. Bohl S, Wassmuth R, Abdel-Aty H, Rudolph A, Messroghli D, Dietz R, et al. Delayed enhancement cardiac magnetic resonance imaging reveals typical patterns of myocardial injury in patients with various forms of non-ischemic heart disease. Int J Cardiovasc Imaging. 2008;24:597-607.

25. Deb K, Djavidani B, Buchner S, Poschenrieder F, Heinicke N, Feuerbach S, et al. Time course of eosinophilic myocarditis visualized by CMR. J Cardiovasc Magn Reson. 2008;10:21.

110 / Brazilian Journal of Medicine Health. 2013 Ago; 2(1):99-110 